\title{
THE PROBLEM OF FERTILIZATION*
}

BY

\section{LORD ROTHSCHILD}

Fertilization may be defined as the incitement of the egg to development by a spermatozoon and the conveyance of paternal qualities to the egg (Wilson, 1925). The central problem in fertilization is: What is it that the spermatozoon does to the egg which transforms it from being an inert and somewhat moribund cell into a highly organized and differentiated embryo?

\section{Morphology of Fertilization}

Usually the egg is a fairly large cell, in the case of human beings about the size of a small pin's head; this large size is often due to the presence of nutrient material within the cell. The spermatozoon is usually extremely small. It consists of a head entirely composed of nuclear material, a middle piece, the functions of which are not clearly understood, and a vibratile tail. The relative sizes of a human egg and the head of a human spermatozoon are about those of a football and a marble.

The main characteristics of the fertilization reaction are : (1) Both eggs and spermatozoa must be ripe for fertilization to take place. (2) Fertilization is specific; that is to say, crosses between different species or genera can rarely be achieved, or, if they are, the chances of a normal embryo resulting are small. (3) Fertilization is an irreversible phenomenon in nature. (4) Only one spermatozoon fertilizes an egg. (5) After adhesion of the spermatozoon to the egg surface a membrane, the fertilization membrane, is thrown off from this surface. (6) The head of the spermatozoon rotates through 180 degrees when it has passed through the egg surface. (7) The head of the spermatozoon within the egg and the nucleus of the egg travel through the cell on somewhat curved and different paths to meet in about the centre of the egg. Then fertilization merges into cell division and development.

In this brief description there are of course various omissions and generalizations to which there are exceptions. For example, some eggs are fertilized while unripe, while it is common knowledge that interspecific crosses are possible. Even polyspermy occurs on occasion without pathological results. Nevertheless, one must assume that these morphological characteristics are significant and, as such, are particularly worthy of investigation.

The first part of the fertilization reaction occurs immediately after the spermatozoon has adhered to the egg surface. It is called activation, and involves the prevention of other spermatozoa from getting in (the block to polyspermy), the elevation of the fertilization membrane, and the irreversibility of the reaction. F. R. Lillie (1912) centrifuged $\in$ ggs of the marine worm Nereis after the spermatozoa had adhered to their surfaces, the centrifugal force tearing the spermatozoa away from the eggs. Nevertheless, the activation changes referred to above all took place, and chromosomes even started to become visible in the female nucleus.

\footnotetext{
*Annual oration to the London Jewish Hospital Medical Society, given on April 17.
}

\section{Permeability and the Biophysics of Fertilization}

It would be surpising if unfertilized and fertilized eggs were in the same physiological condition, and one way of investigating what differences, if any, exist between them is by cbserving the rate at which various substances diffuse in or out of the egg through the egg surface or plasma membrane. For many years there has been a tendency to consider the unfertilized egg as being in an inhibited condition, a condition which is rectified or corrected by fertilization. After fertilization we might expect such alterations in the egg surface as would permit a greater degree of interaction between the egg and its environment. This view received some support from $\mathbf{R}$. S. Lillie (1909), who believed that there might be a similarity between the effects of stimulating an egg by a spermatozoon or parthenogenetic agent and the effects of stimulating a nerve or muscle. One of the characteristics of stimulated nerve and muscle is a temporary increase in the permeability of their cell surfaces, and R. S. Lillie (1917) found the same after fertilization in sea-urchin eggs. This egg swells osmotically when immersed in diluted sea-water, and the amount of water which enters it can be measured. Lillie found that though the total amount of water which enters an egg from sea-water of known hypotonicity is the same before and after fertilization, the rate at which osmotic equilibrium is established is significantly increased by fertilization. This means that though the osmotic properties of the egg as a whole are unaffected by fertilization some change in the egg surface occurs which enables water to pass through more easily. Though sparingly ionized substances, such as water, can pass through the surface more easily after fertilization than before, it does not follow that ions, which are electrically charged, will do the same, though it is an increase in the permeability to ions which is associated with nerve and muscle reactivity. Consequently there is no reason to expect from Lillie's results that fertilization will necessarily be associated with relatively large changes in the electrical properties of the egg surface. Neither the resistance to electric current nor the voltage, if any, across the membrane need be significantly affected. The same applies to the electrical capacity, though there is a large increase in the electrical capacity of the sea-urchin plasma membrane after fertilization (Cole, 1938). Such an increase in capacity might be caused by this membrane becoming thinner, and it is interesting that, on entirely different grounds, other research workers investigating the effect of fertilization on the permeability of sea-urchin eggs to sparingly ionized substances tentatively came to the same conclusion (Stewart and Jacobs, 1936).

At the same time it has been shown on several occasions that during the fertilization reaction acid diffuses out of the sea-urchin egg (Ashbel, 1929; Borei, 1933; Runnström, 1933 ; Laser and Rothschild, 1939). The acid, which so far has not been identified, is a fairly strong one, and it might be expected that its diffusion out of the 
egg would be accompanied by appreciable electrical changes, though these would be very difficult to measure in small eggs. Several attempts have been made to measure such electrical changes, but so far the technical difficulties have proved to be so formidable that the results have been inconclusive (Rothschild, 1938 ; Gelfan, 1931).

The subject of possible analogies between the response of nerve or muscle to a stimulus, and that of the egg to a spermatozoon, is closely bound up with the observation that normally only one spermatozoon fertilizes an egg. What sort of change is propagated through or round the egg from the point of attachment of the fertilizing spermatozoon which prevents other spermatozoa getting in, and what manifestations of this change can be observed?

The rate of propagation of this change must be relatively fast, because a second spermatozoon cannot fertilize an egg some two seconds after the first one has initiated the fertilization reaction. This means, in the case of a seaurchin egg, that we can perhaps set a lower limit for the speed with which this change is propagated in the egg. It is unlikely to be much slower than one millimetre per second and may be much faster. This block to polyspermy is thought to be effected long before the fertilization membrane has lifted away from the egg surface, and probably before the breakdown or dissolution of granules in the egg surface, which takes about ten seconds to be completed at room temperature and which is probably associated with the fertilization membrane reaction (Moser, 1939).

Are these changes, which occur during the fertilization reaction, of general incidence, or are they peculiar to seaurchin eggs, upon which a great deal of the experimental work on fertilization and parthenogenesis is done? In no other eggs has the possibility of acid production on fertilization been investigated. On the other hand, experiments have been done on the permeability of eggs to water before and after fertilization. There is no significant increase in the rate of water penetration into the egg of the annelid worm Chaetopterus pergamentaceus after fertilization (Shapiro, 1939). Similarly there is no increase in oxygen consumption after fertilization in this egg as there is in that of the sea-urchin. Actually the rate of oxygen consumption decreases (Whitaker, 1933). It must follow that investigations on the effect of fertilization or parthenogenetic activation in altering the permeability of the egg surface to water or other sparingly ionized substances are unlikely to help in finding common denominators in the fertilization reaction throughout the animal kingdom; though this does not of course detract from their interest in the wider field of elucidating the structure of the cell membrane. This makes the investigation of other eggs from the point of view of acid production during fertilization one of the most urgently required experiments in this field.

\section{Hyaluronidase}

There may be about $400,000,000$ spermatozoa in a human ejaculation for the fertilization of one, or perhaps two, eggs. The number of spermatozoa can be reduced a certain amount by dilution, but far less than might be expected considering the number of eggs which will be fertilized. It used to be thought that the great number of spermatozoa was necessary to make the probability of fertilization very high. In recent years it has been found that this is by no means the complete explanation, and the discovery that other factors are involved may well have a profound effect on our understanding of the fertilization reaction and also on the treatment of sterility. These discoveries have so far been restricted to mammalian spermatozoa, but they may have some bearing on fertilization in lower organisms, on which so much experimental work has to be carried out. When freshly ovulated, mammalian eggs are surrounded by follicle cells, and it has recently been shown that mammalian testes and spermatozoa contain an enzyme, hyaluronidase, which disperses these follicle cells, or dissolves the substances that make them stick to the egg. It is obviously difficult, if not impossible, for a spermatozoon to come into contact with the egg while the latter is surrounded by these follicle cells, and they must therefore be got rid of before fertilization can take place. The fertilizing spermatozoon is not necessarily among the first lot of spermatozoa which reach the mammalian egg. Their hyaluronidase disperses the follicle cells, leaving the egg bare for subsequent spermatozoa to achieve fertilization. One reason that so large a number of spermatozoa is necessary for successful fertilization is associated with the provision of an adequate concentration of hyaluronidase to disperse the follicle cells. This has been verified by several workers in recent years by artificially inseminating and fertilizing rabbits with suspensions of spermatozoa which were too dilute for successful fertilization under normal conditions, but with hyaluronidase, extracted from dead spermatozoa, added to the sperm suspensions (McClean and Rowlands, 1942). Women have been artificially inseminated, and successful fertilization has resulted, by adding hyaluronidase (extracted from bulls' testes) to semen which previously did not cause fertilization (Leonard and Kurzrok, 1945). Various workers in this field have shown that the amount of hyaluronidase in human semen is proportional to the number of spermatozoa present, which confirms what has been said above about the vital role of this enzyme in mammalian fertilization (Joell and Eichenberger, 1945; Werthessen et al., 1945).

Perhaps more interesting still is the fact that female rats can be immunized against bull testes' hyaluronidase and the immune serum prevents the eggs being denuded of their follicle cells in vitro. Furthermore, normal rat semen itself contains appreciable amounts of an inhibiting substance (Leonard and Kurzrok, 1945). There are two implications to this discovery. First, it may be that new methods of contraception may be developed by the use of immunized blood sera ; secondly, certain cases of sterility may be due to the inhibiting effect of blood on this enzyme's activity. It is know that the bleeding which is occasionally produced during the dilatation of the cervix at the time of artificial insemination is detrimental to fertilization. Clinical experience has also shown that after normal coitus women not infrequently fail to conceive when bleeding from lesions of the cervix or uterus. It therefore seems possible that certain types of sterility previously attributed to a hypothetical spermatozoon immunity may be due to antibodies formed against hyaluronidase, such antibodies passing from the serum into the uterine fluid and preventing fertilization.

The implications of these discoveries are by no means clear when one considers that the eggs of lower organisms, such as sea-urchins, do not have follicle cells round them. Nevertheless, the number of spermatozoa per egg is still enormous. Many eggs have protein coverings round them. For example, frogs' eggs and sea-urchin eggs when laid. are surrounded by a shell of jelly. It might be thought that spermatozoa are so small that they can easily get through barriers of this type, but it is not impossible that the enzyme hyaluronidase plays some part in such cases by facilitating the passage of the spermatozoon through the jelly surrounding the egg. Moreover, there is a species-specific lysin in certain mollusc spermatozoa that dissolves the protein membranes round mollusc eggs (Tyler, 1939a).

In other types of eggs, such as those of the trout, there is a special channel in the outer covering of the egg through which the spermatozoon goes to effect fertilization. Even 
in such cases an enzyme or lysin of the hyaluronidase type might play a part. Spermatozoa are usually thought to bore their way actively into eggs, but in general this does not seem to be the case. The spermatozoon is often passively absorbed, once it adheres to the egg surface. It is not difficult to imagine that an enzyme of the hyaluronidase type might be necessary for the spermatozoon to pass through the protein surface of the egg.

\section{Fertilizin}

One of the most interesting but complicated phenomena associated with the fertilization reaction, at any rate in marine invertebrate eggs, is the appearance of a substance known as fertilizin in the medium round unfertilized eggs. The existence of this substance was first demonstrated by F. R. Lillie (1913), and subsequent researches have shown that it has two main properties. First, suspensions of spermatozoa maintain a higher rate of metabolism and mechanical activity in the presence of these "egg secretions" than in sea-water in which they are not present. Secondly, in the presence of fertilizin spermatozoa come together in clusters which cannot be disrupted by shaking, but which disintegrate spontaneously. The phenomenon is known as agglutination. The agglutinating substance in fertilizin can be used up by spermatozoa, the amount of it in the sea-water round the eggs depending on the number of spermatozoa agglutinated.

In recent years new and important, work has been done on fertilizin, in particular by Tyler and his colleagues. Tyler has advanced the theory that the jelly which is found round unfertilized sea-urchin eggs may be fertilizin, and he has produced evidence confirming this. Tyler (1941) has also shown that fertilizin is not essential for fertilization if the presence or absence of fertilizin is judged by the agglutination or non-agglutination of spermatozoa. If the jelly surrounding the eggs of the sea-urchin Strongylocentrotus purpuratus is removed and the eggs are washed until there is no sperm agglutination whatsoever in the seawater round the eggs, the eggs can nevertheless be fertilized with complete success. It is, however, clear that more spermatozoa are needed when the agglutinating substance has been removed, and this suggests that, apart from the agglutinating effect of fertilizin, the sperm-activation effect referred to earlier on has a function in the fertilization reaction. By increasing the activity of the spermatozoa this substance might exert its influence by increasing the probability of any particular spermatozoon reaching and fertilizing the egg, thus reducing the total number of spermatozoa necessary to ensure a high probability of successful fertilization.

The phenomenon of sperm agglutination presents a further problem of great interest and complexity. This effect is spontaneously reversible (F. R. Lillie, 1913); but after agglutinating spermatozoa markedly lose their capacity to fertilize eggs even though their metabolism and mechanical activity are unimpaired (Tyler, 1941). Nor do they agglutinate a second time. This rather suggests that the agglutination of spermatozoa of the same species is a form of false fertilization between spermatozoa instead of between eggs and spermatozoa; or, at any rate, that there is some reaction between the surfaces of the spermatozoa which is sufficiently similar to that which occurs between an egg and a spermatozoon to alter the spermatozoon in an irreversible manner, though in other ways it is still completely normal The idea of the "muzzled" spermatozoon provides an explanation of a phenomenon which anyone who has watched fertilization under the microscope has observed-that spermatozoa can be seen bumping up against the surface of an egg and then swimming away without having adhered and initiated the fer- tilization reaction. Perhaps the spermatozoa which do this are the "muzzled" ones, and have been " muzzled" by the agglutinin on their way through the jelly toward; the egg surface. The " muzzled" sperm concept provides a further reason for the vast number of spermatozoa needed per egg to obtain a high percentage of fertilization if fertilizin in solution acts as a barrier, in that any spermatozoon which combines with it on the way towards an egg cannot then subsequently effect fertilization (Tyler, 1941). Therefore a lot of spermatozoa are needed to get through the fertilizin barrier in the sea-water round the eggs, leaving the way clear for the fertilizing spermatozoa

Kuhn and his collaborators extracted a substance, echinochrome A, from the ripe eggs of the sea-urchin Arbacia pustulosa. This substance increases the activity of spermatozoa and is considered by this group of workers to be the agent in fertilizin responsible for increasing the activity of spermatozoa (Kuhn and Wallenfels, 1939; Wallenfels, 1943), though this claim has been contested by Tyler (1939b). The chemical similarity between echinochrome and vitamin $K_{1}$ may be significant, particularly in view of the latter's role in blood clotting.

\section{Biochemistry of Fertilization}

From the point of view of the fertilization reaction, an egg exists in four different conditions: unfertilized; being fertilized; fertilized; and, of course, dead. We should expect that these morphological states will be reflected in the biochemistry and, in particular, in the metabolism of the cell Consequently Warburg's (1908) discovery that the oxygen-consumption rate of fertilized sea-urchin eggs increases by a factor of six after fertilization was hailed with enthusiasm. Naturally, it was said, the egg must respire at a greater rate when fertilized, because it is an embryo, cleaving and differentiating. In some eggs, however, respiration rates do not change at fertilization; while in others the rate actually goes down (Whitaker, 1933). In any case the oxygen consumption of unfertilized sea-urchin eggs can be increased to the fertilized level by special means such as the addition of pyocyanine, or even perhaps by an increase of temperature, without activation occurring (Korr, 1937; Laser and Rothschild, 1939). The truth is that the mere measurement of oxygen consumption is inadequate as an index of metabolic activity. To investigate the biochemistry of fertilization we must probe more deeply into the fine structure of cellular metabolism and find out the precise chemical nature of the reaction cycles measured by the disappearance of oxygen and the liberation of carbon dioxide. This, of course, applies only in those cases where the reaction depends on the availability of oxygen.

The availability of oxygen is mentioned because some aggs, at any rate, can be fertilized in the absence of oxygen. Activation may therefore be an anaerobic phenomenon. This is confirmed by the fact that activation, including acid production, can take place in the presence of cyanide, which inhibits the oxidative cycle of reactions catalysed by the cytochrome system. If activation is an anaerobic process the possibility arises that the acid which diffuses out of the egg during the fertilization reaction is a product of anaerobic glycolysis. This suggests some interesting experiments, for we know in some detail the chemical reactions which go to make up the anaerobic glycolysis cycle, and also how to cause inhibition at various points in the cycle. Of course there are anaerobic metabolic systems which are entirely different from glycolysis, which starts with the breakdown of carbohydrate and ends with the production of lactic acid or alcohol. An example is a system of the cysteine desulphurase type, which anaerobically splits cysteine into hydrogen sulphide, ammonia (a 
compound containing ammonia is said to be formed by sea-urchin eggs during fertilization: Örström, 1935), and pyruvic acid.

Another line of investigation is to measure the respiratory quotient during activation, technically a very difficult experiment. There is evidence (Laser and Rothschild, 1939) that the respiratory quotient is rather low during activation, which suggests that activation is associated with the breakdown of fats or proteins rather than carbohydrates.

These facts and speculations about metabolism during the early phases of the fertilization reaction suggest that activation may be associated both with anaerobic processes and with aerobic ones involving the breakdown of fats or proteins. The latter may have an inhibitory effect on the former, including acid production. The inhibitory effects of aerobic processes on fermentation are well known, and the possibility of such a Pasteur effect in the fertilization reaction could be investigated by specific Pasteur-effect poisons such as ethyl carbylamine.

So far we have discussed only the fertilization reaction. There is the separate problem whether there is any metabolic difference between unfertilized and actually fertilized eggs. The researches of Runnström (1933) and Korr (1937) have led to the rather attractive theory that fertilization induces a cytochrome type of metabolism, while the unfertilized egg respires through a non-ferrous autoxidizable carrier of the flavin or pyocyanine type, the cytochrome being present but inactivated. The evidence for this theory is too complicated to discuss in detail here, other than to mention that cyanide is stated to have little inhibitory effect on unfertilized eggs, but a marked effect on fertilized ones. How is cytochrome inactivated in the unfertilized egg? It is well known that changes in the physical state of the egg protoplasm take place as a result of fertilization (Mirsky, 1936; Mazia, 1937 ; Monroy and Montalenti, 1946); these might result in the activation of cytochrome, but there is no evidence that they do. Korr and Runnström's results have recently been disputed (Robbie, 1946), and it is therefore urgently necessary to repeat the experiments, which, from a technical point of view, are comparatively simple.

\section{REFERENCES}

Ashbel, R. (1929). Boll. Soc. ital. Biol. sper., 4, 492.

Borei, H. (1933). Z. vergl..Physiol., 20, 258 .

Cole, K. S. (1938). Nature, 141, 79

Gelfan, S. (1931) Proc, Soc, exp. Biol. N.Y., 29, 58.

Joël, C. A., and Eichenberger, E. (1945). Schweiz. med. Wschr., 75, 601 .

Korr, I. M. (1937). J. cell. comp. Physiol., 10, 461.

Kuhn, R., and Wallenfels, K. (1939). Ber. dtsch. chem. Ges., 72, 1407

Laser, H., and Rothschild, Lord (1939). Proc. roy. Soc., B., 126, 539. Leonard, S. L., and Kurzrok, R. (1945). Endocrinology, 37, 171.

Lillie, F. R. (1912). J. exp. Zool., 12, 413.

Lillie, F. R. (1912). 14. exp.

Lillie, R. S. (1909). Biol. Bull., 17, 188.

- (1917). Amer. J. Physiol., 43, 43.

Mazia, D. (1937). J. cell. comp. physiol., 19, 291

McClean, D., and Rowlands, I. W. (1942). Nature, 150, 627.

Mirsky, A. E. (1936). Science, 84, 333.

Monroy, A and Montalenti, G. (1946) Nature, 158, 239.

Moser, F. (1939). J. exp. Zool., 80, 423

Örström, А. (1935). Ark. Zool., 28в, No. 6

Robbie, W. A. (1946). J. cell. comp. Physiol., 28, 305.

Rothschild, Lord (1938). J. exp. Biol., 15, 209.

Runnström, J. (1933). Biochem. Z., 258, 257.

Shapiro, H. (1939). Biol. Bull., 77, 317.

Stewart, D. R., and Jacobs, M. H. (1936). J. cell. comp. Physiol., 7, 333 .

Tyler, A. (1939a). Proc. nat. Acad. Sci., 25, 317

2 (1939b). Ibid., 25, 523. (1941). Biol. Bull, 81, 190

Wallenfels, K. (1943). Ber. dtsch. chem. Ges., 76, 323.

Wallenfels, K. (1943). Ber. dtsch. chem. Ges.,

Werthessen, N. T., Berman, S., Greenberg, B. E., and Gargill, S. L. (1945). J. Urol., 54, 565.

Whitaker, D. M. (1933). J. gen. Physiol., 16, 475.

Wilson, E. B. (1925). The Cell in Development and Heredity. New York.

\section{INFLUENCE OF PENICILLIN ON THE COAGULATION OF BLOOD}

\section{WITH ESPECIAL REFERENCE TO CERTAIN DENTAL OPERATIONS}

BY

\section{Sir ALEXANDER FLEMING, F.R.S. F.R.C.P., F.R.C.S.}

Professor of Bacteriology, University of London; Director, Inoculation Dept., St. Mary's Hospital

AND

\author{
E. W. FISH, M.D., D.Sc.
}

Honorary Dental Surgeon, St. Mary's Hospital

Certain minor operations in oral surgery depend for their success on the organization of a firm blood clot in a cavity in bone. This is particularly the case in the operation of apicectomy, where the excision of the end of the tooth root leaves a small but appreciable cavity in the bone covered only with a thin flap of mucoperiosteum. If the clot in this cavity breaks down the sinus which forms leads down to the cut surface of the tooth root and permits it to become infected.

Since penicillin has been available it has become a common practice to introduce the powder into the cavity before suturing. Such an amount of powder may, however, represent as much as 10,000 units or more of penicillin, and a marked tendency for the clot to break down has been observed in cases so treated. Because of this, experiments were made to see whether penicillin had any action on the coagulation time of blood or on the contraction of the clot.

The first experiment was carried out with an impure commercial penicillin (sodium salt) containing 361 units per mg. Of this, 100,000 units was dissolved in $0.5 \mathrm{ml}$. of normal saline, and serial dilutions $(0.25-\mathrm{ml}$. volumes) were made in normal saline, leaving one volume of normal saline as a control. Normal human blood was then taken from a vein and immediately $0.25-\mathrm{ml}$. volumes were added to each tube and mixed. The tubes were then observed and the time which elapsed before clotting took place was recorded. A later examination was made to see whether or not the clot had contracted.

It was thought that the anticoagulant effect observed might be due to the impurities in the penicillin, so the same experiment was repeated using pure crystalline sodium salt of penicillin, and in the accompanying table the results are

Table showing effect of Penicillin on Coagulation Time of Human Blood

\begin{tabular}{|c|c|c|}
\hline \multirow{2}{*}{$\begin{array}{l}\text { Final Concentration } \\
\text { of Penicillin in Blood } \\
\text { (Units per ml.) }\end{array}$} & \multicolumn{2}{|c|}{ Coagulation Time } \\
\hline & Impure Penicillin & Crystalline Penicillin \\
\hline $\begin{array}{c}50,000 \\
25,000 \\
12,500 \\
6,250 \\
3,125 \\
1,562 \\
781 \\
340 \\
170 \\
\text { Control }\end{array}$ & $\begin{array}{c}\text { No clot in } 24 \text { hours } \\
\text { "75 minutes } \\
2 \text { minutes }\end{array}$ & $\begin{array}{c}\text { No clot in } 24 \text { hours } \\
\text { " } 32 \text { minutes } \\
11 \text { " } \\
7 \\
5 t \text { " } \\
5 \\
2 t ", \\
2 \\
2 \text { " }\end{array}$ \\
\hline
\end{tabular}

contrasted with those obtained with the impure salt. It will be seen that the result with crystalline penicillin differed only in detail from that with the very impure commercial product, showing that penicillin itself has a considerable retarding action on blood-clotting. 